\title{
Pros and Cons of Current Approaches for Detecting Peroxynitrite and Their Applications
}

\author{
Xingmiao Chen ${ }^{1,2,3}$, Hansen Chen ${ }^{1}$, Ruixia Deng ${ }^{1,2}$, Jiangang Shen ${ }^{1,2,3}$
}

\begin{abstract}
Peroxynitrite, a representative of reactive nitrogen species, plays important roles in the physiological and pathological processes of many oxidative stress-related diseases. It is generated from the reaction of nitric oxide $(\mathrm{NO})$ and superoxide $\left(\mathrm{O} 2^{\bullet-}\right)$ and is far more active than its precursors. Peroxynitrite can be further decomposed into other cytotoxic reactive species. Peroxynitrite and its derivatives can interact with various biomolecules such as DNA and proteins. Due to its high reactivity and short lifetime, accurate detection of peroxynitrite in biological systems is a challenge task. In the last decade, huge efforts have been made to develop reliable techniques to assess the generation of peroxynitrite in various cellular and animal experiments. There are three major approaches for peroxynitrite

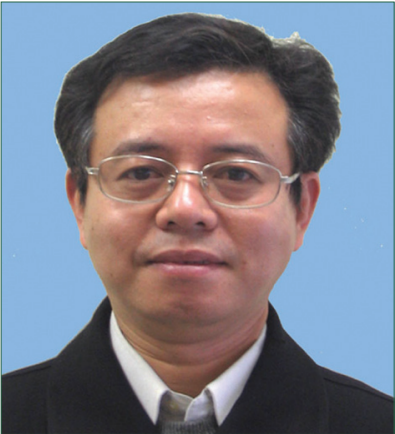

Dr. Jiangang Shen detection, including electrochemical sensors, detection of nitrotyrosine formation, and fluorescent probes. Particularly, progress has been made in developing novel fluorescent probes to detect peroxynitrite with relatively high sensitivity and specificity. Herein, we review the recent progress made in peroxynitrite detection methods and discuss the advantages and disadvantages of these methods. The development of these techniques will offer new opportunities for understanding the roles of peroxynitrite in the oxidative stress-related physiological and pathological conditions and provide platforms for drug discovery targeting peroxynitrite and other free radicals for therapeutic purposes. (Biomed J 2014;37:120-126)
\end{abstract}

Key words: electrochemical sensors, nitrotyrosine, peroxynitrite, probes

$F^{\prime}$ ree radicals are one of the important mediators in the pathological processes of many diseases including cerebral and cardiovascular diseases, diabetes, inflammation diseases, neurodegenerative diseases, cancer, etc., They include reactive oxygen species (ROS) and reactive nitrogen species (RNS). ROS include hydroxyl radical, superoxide, singlet oxygen, hydrogen peroxide, etc., whereas RNS consist of nitric oxide $(\mathrm{NO})$, peroxynitrite $\left(\mathrm{ONOO}^{-}\right), \cdot \mathrm{NO}_{2}$, $\cdot \mathrm{N}_{2} \mathrm{O}_{3}$, etc., Given that ROS research is a relative mature field, much attention has been paid to explore the roles of RNS in these diseases in recent years.

As a representative RNS, $\mathrm{ONOO}^{-}$is considered as a critical cytotoxic factor in oxidative stress-mediated tissue damage. Peroxynitrite is proposed to be the NO toxicity mediator. ${ }^{[2]}$ During oxidative stress, when NO and superoxide anions $\left(\mathrm{O}_{2}^{--}\right)$are simultaneously produced, their reaction is extremely rapid with the diffusion limit to form peroxynitrite $\left(k_{2}=4.7 \times 10^{9} \mathrm{M}^{-1} \mathrm{~s}^{-1}\right)^{[1,2]}$ The reaction rate of $\mathrm{NO}$ and $\mathrm{O}_{2}^{-}{ }^{-}$is much higher than their reactions with other biomeolecules. Peroxynitrite can be further decomposed into other cytotoxic reactive species, such as nitrogen dioxide $\left(\bullet \mathrm{NO}_{2}\right)$, dinitrogen trioxide $\left(\mathrm{N}_{2} \mathrm{O}_{3}\right)$, peroxynitrous acid $(\mathrm{ONOOH})$, hydroxyl and carboxyl radicals, etc., The detrimental effects of $\mathrm{ONOO}^{-}$can be exacerbated by the reaction with $\mathrm{CO}_{2}$ that leads to produce $\mathrm{ONOOC}(\mathrm{O}) \mathrm{O}^{-}$, which further decays into other strong oxidant radicals $\mathrm{NO}_{2}{ }^{-}$and $\mathrm{CO}_{3}{ }^{--}$. Peroxynitrite and its derivates can easily penetrate the cell membrane, and they are far more active than their precursors to induce cytotoxicity. Peroxynitrite and its derivates oxidize multiple

From the ${ }^{1}$ School of Chinese Medicine, LKS Faculty of Medicine, The University of Hong Kong, Hong Kong SAR, China; ${ }^{2}$ Research Centre of Heart, Brain, Hormone and Healthy Aging, the University of Hong Kong, Hong Kong SAR, China; ${ }^{3}$ The University of Hong Kong-Shenzhen Institute of Research and Innovation (HKU-SIRI), China

Received: Feb. 05, 2014; Accepted: May. 14, 2014

Correspondence to: Dr. Jiangang Shen, School of Chinese Medicine, The University of Hong Kong, Hong Kong SAR, China. 10 Sassoon Road, Hong Kong SAR, China. Tel: 852-25890429; Fax: 852-21684259; E-mail: shenjg@hkucc.hku.hk

DOI: $10.4103 / 2319-4170.134084$ 
target molecules either directly or through the secondary generation of highly reactive radicals, resulting in structural modification and dysfunctions in lipids, proteins, and nucleic acids with significant cytotoxic consequences. Peroxynitrite can disrupt DNA integrity, impair the activity of ion channel, ${ }^{[3]}$ break down mitochondrial respiratory chain,${ }^{[4]}$ and even induce cell death. ${ }^{[5]}$ It mediates nitration of tyrosine and cysteine residues in proteins which is one of the crucial pathways contributing to its cytotoxicity. ${ }^{[6]}$ The reaction can induce hydroxylation, peroxidation, and nitration of the proteins and nucleotides, break DNA structure, and induce cell death. ${ }^{[6,7]}$ Peroxynitrite participates in multiple cellular signal transduction pathways and many oxidative stress-related human diseases ${ }^{[2]}$ For example, peroxynitrite-poly (adenosine diphosphate-ribose) polymerase (PARP) pathway is a crucial pathway inducing cell death. The DNA strand break mediated by peroxynitrite activates PARP, and excessive PARP activation leads to depletion of its substrates NAD+ and ATP, inducing apoptosis during ischemic brain injury. ${ }^{[8]}$ On the other hand, $\mathrm{ONOO}^{-}$also takes part in many physiological functions in biological systems. Under some circumstances, high concentration of $\mathrm{ONOO}^{-}$shows anti-viral, anti-microbial, and anti-parasitic activities, whereas low concentration of $\mathrm{ONOO}^{-}$stimulates protective mechanisms in the cardiovascular, nervous, and respiratory systems, ${ }^{[9]}$ and serves as a potential player in the regulation of cell growth. ${ }^{[10]}$

Since $\mathrm{ONOO}^{-}$plays diverse roles in biological systems, development of techniques for monitoring and detecting $\mathrm{ONOO}^{-}$is very important. However, it is a challenging task as the lifetime of $\mathrm{ONOO}^{-}$is very short $(\sim 10 \mathrm{~ms})$ and sometimes $\mathrm{ONOO}^{-}$is present only in trace amounts. Lack of specific and direct detection methods is a drawback that limits the enthusiasm in the studies on $\mathrm{ONOO}^{-}$bioactivities. To resolve the problem, last decade, tremendous efforts were made to develop reliable methods to assess the production of $\mathrm{ONOO}^{-}$in various biological systems. Progress has been made in developing specific and sensitive methods for $\mathrm{ONOO}^{-}$detection recently. Subsequently, many measuring strategies based on different principles have been applied for peroxynitrite detection, such as electrochemical sensors, nitrotyrosine detection, and chemiluminescent/fluorescent probes. Development of these techniques provides new platforms for studying the roles of $\mathrm{ONOO}^{-}$in various cellular and animal experiments and screening the active compounds targeting $\mathrm{ONOO}^{-}$. In this mini review, we briefly evaluate the advantages and disadvantages of the current techniques available for $\mathrm{ONOO}^{-}$detection and discuss their potential applications in biological systems.

\section{Development of electrochemical sensors for peroxynitrite detection}

Several electrochemical ultramicrosensors, including manganese phthalocyanine modified electrode ${ }^{[11]}$ and manganese-[poly-2,5-di-(2-thienyl)-1H-pyrrole)-1 -(p-benzoic acid)] (Mn-pDPB) complex ${ }^{[12]}$ have been developed for detecting $\mathrm{ONOO}^{-}$in both chemical and biological systems. Manganese phthalocyanine electrode was first reported more than 10 years ago. In its design, amperometry peroxynitrite ultramicrosensors (UMS) were fabricated and constructed by electropolymerizing inorganic macromolecular film of tetraaminophthalocyanine manganese (II) and coating chemically with poly (4-vinylpyridine). The detection of peroxynitrite was based on the electrocatalytic reduction of $\mathrm{ONOO}^{-}$. Under optimum conditions, the UMS showed high selectivity and sensitivity to $\mathrm{ONOO}^{-}$determination with a calculated detection limit of $1.8 \times 10^{-8} \mathrm{M}(\mathrm{S} / \mathrm{N}=3) \cdot{ }^{[11]}$ Another electrochemical sensor is Mn-pDPB complex which was applied in a rat plasma sample for the detection of spiked concentrations of $\mathrm{ONOO}^{-}{ }^{[12]}$ The Mn - pDPB complex could selectively enhance the reduction process of $\mathrm{ONOO}^{-}$and was used as the analytical signal for chronoamperometric detection. A polyethyleneimine (PEI) layer was coated on the complex surface to increase selectivity and stability. The chronoamperometric calibration plot showed the hydrodynamic range of $2.0 \times 10^{-8}$ to $5.0 \times 10^{-7} \mathrm{M}$. The detection limit was determined to be $1.9( \pm 0.2) \times 10^{-9} \mathrm{M}$ based on $\mathrm{S} / \mathrm{N}=3 .^{[12]}$ Nevertheless, lack of specificity and fouling of the biosensor are the disadvantages of these sensors due to which they are seldom being used in $\mathrm{ONOO}^{-}$detection. Recently, a simpler and convenient technique based on carbon microfiber electrodes for detecting $\mathrm{ONOO}^{-}$has been reported. ${ }^{[13]}$ The layered composite films of poly (3,4-ethylenedioxythiophene) (PEDOT) and hemin (iron protoporphyrin IX) were used as a platform for amperometric measurement of $\mathrm{ONOO}^{-}$. The electrocatalytic oxidation of $\mathrm{ONOO}^{-}$was characterized by cyclic voltammetry to investigate the intrinsic catalytic role of hemin-electropolymerized thin films on carbon electrodes. The catalytic current increased as a unit to measure the concentration of $\mathrm{ONOO}^{-}$in which a peak potential shifts positively with $\mathrm{ONOO}^{-}$concentration. However, the peak potential of the catalytic oxidation was found to be $\mathrm{pH}$ dependent. The electrochemical detection of peroxynitrite may not be suitable for the studies on disease models which generally have changes in $\mathrm{pH}$ value. Its specificity and sensitivity for peroxynitrite should be justified as well.

\section{Detection of nitrotyrosine formation as a biomarker of peroxynitrite}

Since tyrosine could be nitrated by $\mathrm{ONOO}^{-}$to form 3-nitrotyrosine (3-NT), 3-NT is the most commonly used $\mathrm{ONOO}^{-}$biomarker in biological systems. Several quantification and semi-quantification methods for 3-NT have been developed, such as high-performance liquid chromatogra- 
phy (HPLC) with or without mass spectrometry (MS) for quantification analysis and enzyme-linked immunosorbent assay (ELISA) or immunoblot and immunostaining for semi-quantification. ${ }^{[14,15]}$

Nitrotyrosine formation is considered as a representative biomarker for understanding $\mathrm{ONOO}^{-}$-induced cytotoxicity and nitrosative stress-associated pathological index in many diseases, including diabetes and diabetic complications, ${ }^{[16,17]}$ cerebral ischemia-reperfusion injury, ${ }^{[18]}$ myocardial ischemia-reperfusion injury, ${ }^{[19]}$ neurodegenerative diseases,${ }^{[20]}$ inflammatory lung diseases, ${ }^{[21]}$ etc., For example, by detecting nitrotyrosine, increased $\mathrm{ONOO}^{-}$formation has been reported both in experimental diabetic animal models and in diabetic patients. ${ }^{[22,23]}$ Hyperglycemia induced increased nitrotyrosine formation in the artery wall of monkeys ${ }^{[2]}$ and in diabetic patients. ${ }^{[24,25]}$ The level of nitrotyrosine was also found to be remarkably increased in plasma extracted from type 2 diabetic patients. ${ }^{[26]}$ The increased nitrotyrosine immunoreactivity was correlated with fasting blood glucose, endothelial dysfunction, and increased levels of glycated hemoglobin (HbA1c), intracellular adhesion molecule, and vascular cellular adhesion molecule in the microvasculature of type 2 diabetic patients. ${ }^{[27]}$ Ischemia stroke is another example. Tyrosine nitration was not only used as a footprint of $\mathrm{ONOO}^{-}$in the ischemic brain sections in experimental cerebral ischemia-reperfusion models, ${ }^{[28]}$ but also applied as an evidence of peroxynitrite-mediated brain damage in human brain samples from the patients who died of stroke. ${ }^{[29]}$ Recently, we used 3-NT as a biomarker for evaluating the drug candidates for preventing and treating $\mathrm{ONOO}^{-}$-mediated brain damage in cerebral ischemia-reperfusion injury. ${ }^{[30]}$ Nevertheless, the reliability of using 3-NT as an $\mathrm{ONOO}^{-}$biomarker has been challenged in several aspects. For example, the complicated sample handling procedures and peroxide-containing analytical reaction condition in HPLC/MS could lead to artificial generation of $\mathrm{ONOO}^{-}$and overestimation of $3-\mathrm{NT}^{[31,32]}$ The quality of 3-NT antibody affects the experimental results of immunoblot and immunostaining analysis. In many cases, the expression level of 3-NT in the samples is too low to be detected. More importantly, 3-NT formation can be also induced by other RNS, such as $\mathrm{NO}, \bullet \mathrm{NO}_{2}$, and $\bullet \mathrm{N}_{2} \mathrm{O}_{3}$. The nitrotyrosine formation may not be necessary to be consequence of peroxynitrite-mediated nitration. Thus, detecting 3-NT is not specific for $\mathrm{ONOO}^{-}$. Moreover, it is impossible to monitor real-time formation of $\mathrm{ONOO}^{-}$with this method.

\section{Development of fluorescent probes and sensors for peroxynitrite}

Fluorescent probes have the advantages for monitoring $\mathrm{ONOO}^{-}$with relatively high sensitivity and the capability of affording real-time spatial imaging. Recent progress has enabled monitoring $\mathrm{ONOO}^{-}$generation in living cells by using fluorescent probes. Currently, many fluorescent probes with different fluorophores have been designed and tested in chemical and biological systems.

Dihydrorhodamine (DHR) and dichlorodihydrofluorescein (DCHF) are two typical reduced fluorophores which have been used in more than 2000 studies. ${ }^{[33,34]}$ Peroxynitrite can oxidize these probes to generate fluorescent compounds. DHR has been used to detect RNS including $\mathrm{ONOO}^{-}$and its decomposition products, with $\mathrm{ONOO}^{-}$probably oxidizing DHR to rhodamine $(\mathrm{Rh})$ through radical products rather than directly. However, lack of specificity is the greatest drawback of these probes. Apart from RNS, DHR can also form fluorescent rhodamine efficiently when it reacts with hydroxyl radical, nitric dioxide, carbonate radical, and hypochlorous acid. ${ }^{[35,36]}$ Like DHR, DCHF could also be oxidized by hydroxyl radical, nitric dioxide, carbonate radical, $\mathrm{Fe}$ (II), Fe (III)/ascorbate, cytochrome c, xanthine oxidase, and hypochlorous acid to produce a fluorescent signal. ${ }^{[37]}$ In addition, 2-[6-(4'-hydroxy) phenoxy-3H-xanthen-3-on-9-yl] benzoic acid (HPF) and 2-[6-(4'-amino) phenoxy-3H-xanthen-3-on-9-yl] benzoic acid (APF) were developed for detecting $\mathrm{ONOO}^{-}$, but they undergo cross-reactions with hydroxyl radical and hypochlorous acid and cannot separate peroxynitrite from the hydroxyl radical and hypochlorous acid. ${ }^{[38]}$

Boronate-containing fluorescent probes have been designed for $\mathrm{ONOO}^{-}$detection since they can react rapidly and directly with $\mathrm{ONOO}^{-}$to convert weakly fluorescent arylboronates to strong fluorescent products. ${ }^{[3,40]}$ The reaction rate of boronates with $\mathrm{ONOO}^{-}$is 200 times faster than the rate with hypochlorous acid and a million times faster than with $\mathrm{H}_{2} \mathrm{O}_{2}{ }^{[39]}$ They appear to be better than DHR and DCHF. However, it is still difficult to use boronate-based probes to distinguish $\mathrm{ONOO}^{-}$from $\mathrm{H}_{2} \mathrm{O}_{2}$ since they can be also used to detect $\mathrm{H}_{2} \mathrm{O}_{2} \cdot{ }^{[41,42]}$

Why is the detection of $\mathrm{ONOO}^{-}$so difficult? The reasons can be summarized as the following: (a) Its short lifetime, (b) difficulty in distinguishing it from other ROS or RNS, and (c) no specific endogenous targets. To resolve those problems, a novel genetically encoded probe named boronic acid-derived circularly permuted green fluorescent protein (pnGFP) has been synthesized. A boronic acid moiety has been site-specifically introduced into circularly permuted fluorescent proteins. By examining different protein templates followed by site-targeted random mutagenesis, the protein can be used as a selective $\mathrm{ONOO}^{-}$sensor and thus used for imaging $\mathrm{ONOO}^{-}$which is essentially unresponsive to other common cellular redox signaling molecules in chemical and cellular systems. ${ }^{[43]}$ However, the large molecular weight of this protein-based probe has poor membrane permeability and cannot be used for in vivo 
animal studies. Recently, we have enrolled in developing novel fluorescent probes. A specific reaction between ketone 1 and $\mathrm{ONOO}^{-}$rather than with the other ROS and RNS has been found in the biological system. On the basis of this reaction, a new fluorescent probe HKGreen-1 has been successfully developed, which is highly selective for the detection of $\mathrm{ONOO}^{-}$in living cells. Without peroxynitrite, the dichlorofluorescein part is masked and the probe is nonfluorescent. However, upon reaction with $\mathrm{ONOO}^{-}$, the fluorophore is released, resulting in strong enhancement in fluorescence intensity. ${ }^{[44]}$ With this probe, we visualized endogenous $\mathrm{ONOO}^{-}$generation in oxygen-glucose deprivation cortical neurons. ${ }^{[45]}$ Following this, several new-generation probes such as HKGreen-2 and HKGreen-3 have been developed. ${ }^{[46,47]}$ HKGreen- 2 is modified from HKGreen- 1 based on a similar reaction for better imaging quality, whereas HKGreen-3 is designed on the basis of the rhodol scaffold and the $\mathrm{ONOO}^{-}$-specific oxidation reaction. Both of them show high sensitivity and selectivity for detecting $\mathrm{ONOO}^{-}$in chemical and biological systems.

In addition, several promising fluorescent probes with relatively high sensitivity and selectivity for $\mathrm{ONOO}^{-}$have been recently developed, which are based on different chemical reactions and principles. $\mathrm{PN}_{600}$ was rationally designed on the basis of a unique fluorophore assembly approach. $\mathrm{PN}_{600}$ is a green-emitting coumarin derivative. Upon oxidation by $\mathrm{ONOO}^{-}, \mathrm{PN}_{600}$ is transformed into a highly fluorescent red-emitting resorufin derivative through an orange-emitting intermediate. This three-channel signaling capability enables $\mathrm{PN}_{600}$ to differentiate $\mathrm{ONOO}^{-}$from other ROS and RNS, including hypochlorite and hydroxyl radicals. Moreover, $\mathrm{PN}_{600}$ is membrane permeable and compatible with common tetramethylrhodamine isothiocyanate (TRITC) filter sets, and displays low cytotoxicity. Therefore, $\mathrm{PN}_{600}$ is a promising candidate for in vitro peroxynitrite imaging. ${ }^{[48]}$ Rhodamine B phenyl hydrazide (RBPH) has been recently developed as a novel sensitive and specific $\mathrm{ONOO}^{-}$turn-on fluorescent probe. ${ }^{[49]}$ The probe RBPH can be oxidized by $\mathrm{ONOO}^{-}$which converts RBPH to pink-colored and highly fluorescent Rhodamine B. The $\mathrm{ONOO}^{-}$-induced fluorescent imaging was observed and tested in MCF-7 cells. The fluorescence emission intensity of the Rhodamine B produced in the above process was found to be linearly related to the concentration of $\mathrm{ONOO}^{-}$. The method obeys Beer's law in the concentration range of 2-20 $\mathrm{nM}$ and the detection limit has been found to be $1.4 \mathrm{nM}$. The probe was found to have selectivity to react with $\mathrm{ONOO}^{-}$in the $\mathrm{pH}$ range $6-8$ with cell membrane permeability.

$\mathrm{N}$-(2-aminophenyl)-5-(dimethylamino)-1-naphthalenesulfonic amide (Ds-DAB) is another promising probe for $\mathrm{ONOO}^{-}$detection. ${ }^{[50]}$ Peoxynitrite selectively reacts with Ds-DAB in aqueous solution and results in obvious fluorescence enhancement, but without interference from other ROS and RNS. The advantages of high selectivity, fast reaction rate, and $\mathrm{ONOO}^{-}$imaging render Ds-DAB suitable for $\mathrm{ONOO}^{-}$detection in biological systems. However, their applications remain to be further verified with different animal and cellular experimental systems.

\section{Conclusion and perspectives}

In summary, great efforts have been made to develop new techniques for detecting $\mathrm{ONOO}^{-}$production in biological systems. There are at least three major directions to go for measuring $\mathrm{ONOO}^{-}$, including electrochemical sensors, detection of nitrotyrosine formation, and fluorescent probes [Table 1]. Among those methods, electrochemical sensors are seldom used in literature. Although nitrotyrosine detection is widely applied as a footprint of $\mathrm{ONOO}^{-}$formation and index of nitrosative stress in both cellular and animal experimental systems, the detection of nitrotyrosine is only used as a marker for the consequences of RNS-mediated tissue damage rather than detecting $\mathrm{ONOO}^{-}$itself. The nitration of tyrosine can be due to other RNS in addition to $\mathrm{ONOO}^{-}$, making the interpretation of experimental results very difficult. Thus, in recent years, major work has been done in developing highly selective and sensitive fluorescent probes for imaging $\mathrm{ONOO}^{-}$in both in vitro and in vivo experiments. Nowadays, more and more comprehensive and advanced

Table 1: Pros and cons of currently used common methods for peroxynitrite detection

\begin{tabular}{|c|c|c|}
\hline Methods & Pros & Cons \\
\hline $\begin{array}{l}\text { Electrochemical } \\
\text { sensors, including } \\
\text { UMS, Mn-pDPB } \\
\text { complex, and carbon } \\
\text { microfiber electrodes }\end{array}$ & $\begin{array}{l}\text { Relatively high } \\
\text { sensitivity } \\
\text { Capability of } \\
\text { quantification }\end{array}$ & $\begin{array}{l}\text { Lack of specificity } \\
\text { Fouling of biosensors } \\
\text { pH value dependent }\end{array}$ \\
\hline $\begin{array}{l}\text { Detection of } \\
\text { nitrotyrosine by HPLC, } \\
\text { LC/MS/MS, ELISA, } \\
\text { immunoblotting, and } \\
\text { immunostaining }\end{array}$ & $\begin{array}{l}\text { Most commonly used } \\
\text { Capability of } \\
\text { quantification } \\
\text { Convenient for } \\
\text { in vivo study } \\
\text { Related to disease } \\
\text { damage }\end{array}$ & $\begin{array}{l}\text { Lack of specificity } \\
\text { High detection limit } \\
\text { Affected by } \\
\text { antibody's quality } \\
\text { No real-time } \\
\text { monitoring }\end{array}$ \\
\hline $\begin{array}{l}\text { Fluorescent probes: } \\
\text { Including DHR and } \\
\text { DCHF, APF and HPF, } \\
\text { pnGFP, HKGreen-1,2,3, } \\
\text { PN }_{600}, \text { RBPH, and } \\
\text { Ds-DAB }\end{array}$ & $\begin{array}{l}\text { Relatively high } \\
\text { sensitivity } \\
\text { Capability of } \\
\text { real-time imaging } \\
\text { Simplicity for use }\end{array}$ & $\begin{array}{l}\text { Specificity depends } \\
\text { on different probe } \\
\text { structures } \\
\text { Some probes are pH } \\
\text { dependent } \\
\text { Many probes have not } \\
\text { been verified in vivo }\end{array}$ \\
\hline
\end{tabular}

Abbreviations: UMS: Ultramicrosensors; HPLC: Highperformance liquid chromatography; LC: liquid chromatography; MS: Mass spectrometry; DHR: Dihydrorhodamine; DCHF:

Dichlorodihydrofluorescein; APF: 2-[6-(4'-amino)phenoxy-3Hxanthen-3-on-9-yl]benzoic acid; HPF: 2-[6-(4'-hydroxy)phenoxy-3Hxanthen-3-on-9-yl]benzoic acid; HK: Hong Kong; RBPH: Rhodamine B phenyl hydrazide; Ds-DAB: N-(2-aminophenyl)-5-(dimethylamino)1-naphthalenesulfonic amide; Mn-pDPB: manganese-[poly-2,5-di-(2thienyl)-1H-pyrrole)-1-(p-benzoic acid)] 
imaging facilities and instruments have been applied in both in vitro and in vivo studies. Developing high-throughput assays for real-time monitoring of various RNS and ROS in biological systems is a good direction. Monitoring the diagnostic marker produced from the reaction between specific chemical probes and the oxidant species makes simultaneous assay of these reactive species by using a multiwall plate possible. ${ }^{[51]}$ Detecting the diagnostic biomarkers in plasma has a broad application potential to monitor the disease progress and therapeutic outcome. For example, the concentration of plasma peroxiredoxin, an endogenous antioxidant that functions as a peroxide and peroxynitrite scavenger, was decreased in severe stroke and inversely correlated to the systemic markers of inflammation in acute stroke patients. ${ }^{[52]}$ We can expect that developing real-time spatial imaging probes will have broad application potential in future. However, we must note that the most commonly used probes like DCHF and DHR can provide some information about the redox environment of the cells, but they have cross-reactions with many other ROS and RNS, in addition to $\mathrm{ONOO}^{-}$. The fluorescent imaging can be affected by numerous chemical interactions, and is not simply due to the increased oxidant or nitrosative generation. These are many factors due to oxygen, superoxide, and various antioxidant molecules that can interfere with the fluorescent signal of the probes. In recent years, new generation probes based on different chemical mechanisms and principles appear to be promising and have the potential to detect $\mathrm{ONOO}^{-}$specifically with relatively high sensitivity. However, their values remain to be further tested with a series of experiments. We are at the gate opening to the magic world. As a recent review article remarked, data from oxidant-sensitive fluorescent probes somehow provide information on cellular redox activity, but are widely misinterpreted. Newly developed non-redox probes show promise, but are generally not available, and more information on specificity and cellular reactions is needed. We are yet to have tools that can specifically quantify cellular production of specific oxidants. ${ }^{[53]}$ Moreover, efforts are also made for developing various methods to identify nitration protein and nitrotyrosine site. ${ }^{\left[{ }^{[4,55]}\right.}$ With the development of novel techniques for special assessment of not only $\mathrm{ONOO}^{-}$but also nitration protein and nitrotyrosine sites, we will be able to better understand the oxidation and redox mechanisms in both physiological and pathological conditions. The progress offers new opportunities and platforms for drug discovery targeting peroxynitrite and other free radicals for therapeutic purposes.

\section{Acknowledgment}

This work was supported by Hong Kong General Research Fund (GRF No. 777611M and No. 776512M), Research Grant Council, Hong Kong SAR; Grant from Na- tional Natural Science Foundation of China (No. 31270902); and Seed Funding Programme for Basic Research from The University of Hong Kong (201011159053).

\section{REFERENCES}

1. Radi R, Peluffo G, Alvarez MN, Naviliat M, Cayota A. Unraveling peroxynitrite formation in biological systems. Free Radical Biol Med 2001;30:463-88.

2. Pacher P, Beckman JS, Liaudet L. Nitric oxide and peroxynitrite in health and disease. Physiol Rev 2007;87:315-424.

3. Grover AK, Samson SE. Effect of superoxide radical on Ca-2+ pumps of coronary-artery. Am J Physiol 1988;255:C297-303.

4. Jang B, Han S. Biochemical properties of cytochrome $\mathrm{c}$ nitrated by peroxynitrite. Biochimie 2006;88:53-8.

5. Virag L, Szabo E, Gergely P, Szabo C. Peroxynitrite-induced cytotoxicity: Mechanism and opportunities for intervention. Toxicol Lett 2003;140-141:113-24.

6. Radi R. Nitric oxide, oxidants, and protein tyrosine nitration. Proc Natl Acad Sci U S A 2004;101:4003-8.

7. Moreno JJ, Pryor WA. Inactivation of alpha 1-proteinase inhibitor by peroxynitrite. Chem Res Toxicol 1992;5:425-31.

8. Endres M, Wang ZQ, Namura S, Waeber C, Moskowitz MA. Ischemic brain injury is mediated by the activation of poly (ADP-ribose) polymerase. J Cereb Blood Flow Metab 1997;17:1143-51.

9. Ascenzi P, di Masi A, Sciorati C, Clementi E. Peroxynitrite-An ugly biofactor? Biofactors 2010;36:264-73.

10. Agbani EO, Coats P, Wadsworth RM. Acute hypoxia stimulates intracellular peroxynitrite formation associated with pulmonary artery smooth muscle cell proliferation. J Cardiovasc Pharmacol $2011 ; 57: 584-8$

11. Xue J, Ying X, Chen J, Xian Y, Jin L. Amperometric ultramicrosensors for peroxynitrite detection and its application toward single myocardial cells. Anal Chem 2000;72:5313-21.

12. Koh WC, Son JI, Choe ES, Shim YB. Electrochemical detection of peroxynitrite using a biosensor based on a conducting polymer-manganese ion complex. Anal Chem 2010;82:10075-82.

13. Peteu SF, Bose T, Bayachou M. Polymerized hemin as an electrocatalytic platform for peroxynitrite's oxidation and detection. Anal Chim Acta 2013;780:81-8.

14. Tarpey MM, Wink DA, Grisham MB. Methods for detection of reactive metabolites of oxygen and nitrogen: In vitro and in vivo considerations. Am J Physiol Regul Integr Comp Physiol 2004;286:R431-44.

15. Rabbani N, Thornalley PJ. Assay of 3-nitrotyrosine in tissues and body fluids by liquid chromatography with tandem mass spectrometric detection. Methods Enzymol 2008;440:337-59.

16. Zou MH, Cohen R, Ullrich V. Peroxynitrite and vascular endothelial dysfunction in diabetes mellitus. Endothelium 2004;11:89-97.

17. Pacher P, Obrosova IG, Mabley JG, Szabó C. Role of nitrosative stress and peroxynitrite in the pathogenesis of diabetic complications. Emerging new therapeutical strategies. Curr Med Chem $2005 ; 12: 267-75$

18. Gürsoy-Ozdemir Y, Can A, Dalkara T. Reperfusion-induced 
oxidative/nitrative injury to neurovascular unit after focal cerebral ischemia. Stroke 2004;35:1449-53.

19. Hayashi Y, Sawa Y, Ohtake S, Fukuyama N, Nakazawa H, Matsuda H. Peroxynitrite formation from human myocardium after ischemia-reperfusion during open heart operation. Ann Thorac Surg 2001;72:571-6.

20. Pérez-De La Cruz V, González-Cortés C, Galván-Arzate S, Medina-Campos ON, Pérez-Severiano F, Ali SF, et al. Excitotoxic brain damage involves early peroxynitrite formation in a model of Huntington's disease in rats: Protective role of iron porphyrinate $5,10,15,20$-tetrakis (4-sulfonatophenyl) porphyrinate iron (III). Neuroscience 2005;135:463-74.

21. Sugiura $\mathrm{H}$, Ichinose $\mathrm{M}$. Nitrative stress in inflammatory lung diseases. Nitric Oxide 2011;25:138-44.

22. Pennathur S, Wagner JD, Leeuwenburgh C, Litwak KN, Heinecke JW. A hydroxyl radical-like species oxidizes cynomolgus monkey artery wall proteins in early diabetic vascular disease. J Clin Invest 2001;107:853-60.

23. Zou MH, Shi C, Cohen RA. High glucose via peroxynitrite causes tyrosine nitration and inactivation of prostacyclin synthase that is associated with thromboxane/prostaglandin $\mathrm{H}$ (2) receptor-mediated apoptosis and adhesion molecule expression in cultured human aortic endothelial cells. Diabetes 2002;51:198-202.

24. Ceriello A, Taboga C, Tonutti L, Quagliaro L, Piconi L, Bais B, et al. Evidence for an independent and cumulative effect of postprandial hypertriglyceridemia and hyperglycemia on endothelial dysfunction and oxidative stress generation: Effects of short- and long-term simvastatin treatment. Circulation 2002;106:1211-8.

25. Ceriello A, Quagliaro L, Catone B, Pascon R, Piazzola M, Bais B, et al. Role of hyperglycemia in nitrotyrosine postprandial generation. Diabetes Care 2002;25:1439-43.

26. Ceriello A, Mercuri F, Quagliaro L, Assaloni R, Motz E, Tonutti L, et al. Detection of nitrotyrosine in the diabetic plasma: Evidence of oxidative stress. Diabetologia 2001;44:834-8.

27. Szabo C, Zanchi A, Komjati K, Pacher P, Krolewski AS, Quist WC, et al. Poly (ADP-Ribose) polymerase is activated in subjects at risk of developing type 2 diabetes and is associated with impaired vascular reactivity. Circulation 2002;106:2680-6.

28. Maneen MJ, Hannah R, Vitullo L, DeLance N, Cipolla MJ. Peroxynitrite diminishes myogenic activity and is associated with decreased vascular smooth muscle F-actin in rat posterior cerebral arteries. Stroke 2006;37:894-9.

29. Forster C, Clark HB, Ross ME, Iadecola C. Inducible nitric oxide synthase expression in human cerebral infarcts. Acta Neuropathol 1999;97:215-20

30. Xu M, Chen $\mathrm{X}, \mathrm{Gu} \mathrm{Y}$, Peng $\mathrm{T}$, Yang $\mathrm{D}$, Chang $\mathrm{RC}$, et al. Baicalin can scavenge peroxynitrite and ameliorate endogenous peroxynitrite-mediated neurotoxicity in cerebral ischemia-reperfusion injury. J Ethnopharmacol 2013;150:116-24.

31. Yi D, Ingelse BA, Duncan MW, Smythe GA. Quantification of 3-nitrotyrosine in biological tissues and fluids: Generating valid results by eliminating artifactual formation. J Am Soc Mass Spectrom 2000;11:578-86

32. Frost MT, Halliwell B, Moore KP. Analysis of free and protein-bound nitrotyrosine in human plasma by a gas chromatography/mass spectrometry method that avoids nitration artifacts. Biochem J 2000;345:453-8
33. Crow JP. Dichlorodihydrofl uorescein and dihydrorhodamine 123 are sensitive indicators of peroxynitrite in vitro: Implications for intracellular measurement of reactive nitrogen and oxygen species. Nitric Oxide 1997;1:145-57.

34. Wardman P. Methods to measure the reactivity of peroxynitritederived oxidants toward reduced fl uoresceins and rhodamines. Methods Enzymol 2008;441:261-82.

35. Jourd'heuil D, Jourd'heuil FL, Kutchukian PS, Musah RA, Wink DA, Grisham MB. Reaction of superoxide and nitric oxide with peroxynitrite. Implications for peroxynitrite-mediated oxidation reactions in vivo. J Biol Chem 2001;276:28799-805.

36. Wardman P. Fluorescent and luminescent probes for measurement of oxidative and nitrosative species in cells and tissues: Progress, pitfalls, and prospects. Free Radic Biol Med 2007;43:995-1022.

37. Hempel SL, Buettner GR, O’Malley YQ, Wessels DA, Flaherty DM. Dihydrofluorescein diacetate is superior for detecting intracellular oxidants: Comparison with 2',7'-dichlorodihydrofluorescein diacetate, 5(and 6)-carboxy-2',7'-dichlorodihydrofluorescein diacetate, and dihydrorhodamine 123. Free Radic Biol Med 1999;27:146-59.

38. Setsukinai K, Urano Y, Kakinuma K, Majima HJ, Nagano T. Development of novel fluorescence probes that can reliably detect reactive oxygen species and distinguish specific species. J Biol Chem 2003;278:3170-5.

39. Sikora A, Zielonka J, Lopez M, Joseph J, Kalyanaraman B. Direct oxidation of boronates by peroxynitrite: Mechanism and implications in fluorescence imaging of peroxynitrite. Free Radic Biol Med 2009;47:1401-7.

40. Yu F, Song P, Li P, Wang B, Han K. A fluorescent probe directly detect peroxynitrite based on boronate oxidation and its applications for fluorescence imaging in living cells. Analyst 2012;137:3740-9.

41. Miller EW, Albers AE, Pralle A, Isacoff EY, Chang CJ. Boronate-based fluorescent probes for imaging cellular hydrogen peroxide. J Am Chem Soc 2005;127:16652-9.

42. Zielonka J, Sikora A, Hardy M, Joseph J, Dranka BP, Kalyanaraman B Boronate probes as diagnostic tools for real time monitoring of peroxynitrite and hydroperoxides. Chem Res Toxicol 2012;25:1793-9.

43. Chen ZJ, Ren W, Wright QE, Ai HW. Genetically encoded fluorescent probe for the selective detection of peroxynitrite. J Am Chem Soc 2013;135:14940-3.

44. Yang D, Wang HL, Sun ZN, Chung NW, Shen JG. A highly selective fluorescent probe for the detection and imaging of peroxynitrite in living cells. J Am Chem Soc 2006;128:6004-5.

45. Yang D, Sun ZN, Peng T, Wang HL, Shen JG, Chen Y, et al. Synthetic fluorescent probes for imaging of peroxynitrite and hypochlorous acid in living cells. Methods Mol Biol 2010;591:93-103.

46. Sun ZN, Wang HL, Liu FQ, Chen Y, Tam PK, Yang D. BODIPY-based fluorescent probe for peroxynitrite detection and imaging in living cells. Org Lett 2009;11:1887-90.

47. Peng T, Yang D. HKGreen-3: A rhodol-based fluorescent probe for peroxynitrite. Org Lett 2010;12:4932-35.

48. Zhang Q, Zhu Z, Zheng Y, Cheng J, Zhang N, Long YT, et al. A three-channel fluorescent probe that distinguishes peroxynitrite from hypochlorite. J Am Chem Soc 2012;134:18479-82.

49. Ambikapathi G, Kempahanumakkagari SK, Ramappa Lamani B, Kuramkote Shivanna D, Bodagur Maregowda H, Gupta A, et al. Bioimaging of peroxynitrite in MCF-7 cells by a new fluorescent 
probe rhodamine B phenyl hydrazide. J Fluoresc 2013;23:705-12.

50. Lin KK, Wu SC, Hsu KM, Hung CH, Liaw WF, Wang YM. A N-(2-aminophenyl)-5-(dimethylamino)-1-naphthalenesulfonic amide (Ds-DAB) based fluorescent chemosensor for peroxynitrite. Org Lett 2013;15:4242-5.

51. Zielonka J, Joseph J, Sikora A, Kalyanaraman B. Real-time monitoring of reactive oxygen and nitrogen species in a multiwell plate using the diagnostic marker products of specific probes. Methods Enzymol 2013;526:145-57.

52. Kunze A, Zierath D, Tanzi P, Cain K, Becker K. Peroxiredoxin 5 (PRX5) is correlated inversely to systemic markers of inflammation in acute stroke. Stroke 2014;45:608-10.
53. Winterbourn CC. The challenges of using fluorescent probes to detect and quantify specific reactive oxygen species in living cells. Biochim Biophys Acta 2014;1840:730-8.

54. Díaz-Moreno I, García-Heredia JM, González-Arzola K, Díaz-Quintana A, De la Rosa MÁ. Recent methodological advances in the analysis of protein tyrosine nitration. Chemphyschem 2013;14:3095-102.

55. Seeley KW, Fertig AR, Dufresne CP, Pinho JP, Stevens SM Jr. Evaluation of a method for nitrotyrosine site identification and relative quantitation using a stable isotope-Labeled nitrated spikein standard and high resolution fourier transform MS and MS/MS Analysis. Int J Mol Sci 2014;15:6265-85. 